\title{
EDUCACION PERMANENTE Y CAMBIO SOCIAL
}

Margarita Peña Borrero*

\section{Educación permanente y la reproducción de la desigualdad}

\section{El Problema}

Pese a las profundas implicaciones políticas de un concepto de esta naturaleza, parece existir un acuerdo general acerca del hecho de que la educación no debe limitarse a cierto período en la vida de un individuo porque el aprendizaje no sólo ocurre en la escuela sino en todas las situaciones a través de la vida de una persona. Debido al gran número de problemas cubiertos por tal definición, es necesario limitarla con el fin de hacer posible el debate futuro y evitar confusiones con otros términos 'de moda' en la literatura educativa contemporánea.

Todavía moviéndonos en un espacio neutral, lo que parece ser posible hasta cierto punto, por lo menos como punto de partida, la educación permanente se refiere tanto a los patrones formales y no formales de educación, al aprendizaje planificado así como al aprendizaje incidental. Esto significa romper el monopolio de las instituciones educativas, tradicionalmente las únicas capacitadas para dar educación, y convertir la totalidad de la sociedad en un entorno educativo. Así pues, educación permanente no significa escolarización permanente. Tampoco es lo mismo que educación de adultos, aunque no existe duda sobre su importancia desde la perspectiva de la educación permanente, debido al tradicional aislamiento de los adultos de cualquier proceso educativo usualmente restringidos al angosto marco de la escuela.

Con esta definición surgen dificultades cuando se le da a la educación permanente más y más connotaciones. Definiciones del tipo de las de UNESCO tienden a verla no sólo como una cura para las deficiencias del sistema educativo, sino como un antídoto contra los graves problemas de la sociedad. Su carácter universal conduce a la conclusión, casi automática, de que la educación permanente representa la democratización de la educación y de que su meta última es mantener y mejorar la calidad de la vida ${ }^{35}$.

Estos argumentos parecen estar fuera de discusión. Los problemas aparecen cuando sus implicaciones políticas son examinadas más de cerca. ¿Significa la educación permanente democratización dentro de un sistema socio-económico determinado o es más bien el resultado en una sociedad que ha eliminado las limitaciones que previamente hacían la educación permanente imposible? Si mantener, mejorar la educación de la vida es su meta final ¿cuáles valores merecen conservarse y cuáles deben ser mejorados y en qué dirección?

Interrogantes de esta naturaleza han originado diferentes tendencias dentro de la literatura sobre educación permanente. Ireland ${ }^{36}$ distingue dos corrientes bien diferenciadas. La visión "optimista", de una parte, está caracterizada por su amplia conceptuailización del término y por su pretendida neutralidad. Escritores dentro de esta tendencia se centran principalmente en la elaboración de completos modelos para la

\footnotetext{
${ }^{*}$ Licenciada en Filosofía y Letras. Universidad Javeriana. Master of Arts., Ohio University. Master of Sciences, University of Edinburgh.

${ }^{35}$ Dave.'The Foundations of lifelong Education. pág. 20-22.

${ }^{36}$ Ireland. Gelpis.'View of Lifelong Education. pág. 22. 
educación permanente -incluyendo currículo escolar, administración educativa y una nueva y sofisticada tecnología educativa - pero de una manera teórica que deja sin solución el problema de su implementación y que en la mayoría de los casos ignora el contexto histórico.

Además de su ambigüedad, esta visión de educación permanente es en esencia conservadora, pues supone que la educación permanente se desarrolla en un 'vacío político' lo cual esconde el hecho de que sus defensores ven el sistema socioeconómico como algo dado y que no debe cambiarse sino mejorarse por medio de unas estrategias administrativas y educativas. Este fácil optimismo ha sido severamente atacado por un grupo de teóricos que insisten en el doble potencial de la educación permanente. Para estos últimos, el problema central es si la educación permanente pretende realmente incrementar la participación de todos los grupos de la sociedad en el proceso de toma de decisiones, o por el contrario, generar apoyo para la autoridad establecida ${ }^{37}$. Dicho de otra manera, esto significa que la educación permanente no puede ser considerada como neutral.

Reconocer el carácter político de la educación permanente -y por lo tanto de la educación misma - no representa ninguna novedad ya que las relaciones existentes entre educación y el sistema socioeconómico han sido ya 'descubiertas' y estudiadas por largo tiempo. Pero existen diferentes formas para analizar la naturaleza de esta relación. El rol de la educación dentro de cualquier sociedad depende significativamente de la manera como los educadores y planificadores se vean a sí mismos, ya sea como miembros activos de una sociedad cambiante o como simples productos del sistema establecido. Así pues, aceptar que la educación no es neutral porque está inserta en un determinado sistema socioeconómico puede llegar a convertirse en un arma de doble filo: La educación puede ser vista bien como un medio para reproducir las existentes condiciones de injusticia y desigualdad, o como un factor de cambio. Así pues, rechazar la neutralidad de la educación no es suficiente. Si la educación no es neutral, ¿cuál es entonces su específica función en la sociedad? En las siguientes páginas se discuten diversas posiciones con respecto a este interrogante, todos ellos basados en análisis marxistas de la sociedad y de la cultura.

\section{Educación y reproductivismo: El enfoque de Althusser}

Para existir, cualquier formación social debe generar los medios para reproducir las fuerzas de producción y las relaciones de producción. Tomando esto como su afirmación básica, Althusser ${ }^{38}$ explica por qué la educación es uno de los mecanismos por los cuales la sociedad se reproduce a sí misma. Esta visión se basa, a su vez, en el supuesto de que existen en la sociedad una base determinante y una superestructura determinada cuya relación puede expresarse, según Williams como una "relación espacial defialda y prefijada". No es el propósito de este artículo analizar profundamente los escritos de Althusser, sino examinar brevemente los argumentos relacionados con el rol de la educación en la reproducción de las condiciones sociales y económicas existentes.

El punto de partida de Althusser es la representación de la sociedad como un edificio de dos pisos en el cual el piso de arriba no podría sostenerse si no descansara sólidamente en su base. Esta metáfora es utilizada para ilustrar la naturaleza determinante del piso de

\footnotetext{
${ }^{37}$ Idem, pág. 26.

${ }^{38}$ Althusser "Ideology and the Ideologica.1 State Apparatures", 1971.
} 
abajo - la base económica - en relación con el piso de arriba —la superestructura-y revela en consecuencia el problema crucial del determinismo; a saber, que "es la base la que en última instancia determina todo el edificio". Althuser continúa explicando cómo el carácter descriptivo de esta representación es superado cuando la existencia de la superestructura es concebida en términos de la reproducción de las relaciones de producción. Esta es la acción recíproca de la superestructura sobre la base ${ }^{39}$

Tal como está presentado en ideología y Aparatos Ideológicos del Estado, el Estado, como una de las instancias de la superestructura, posee aparatos cuya meta final es reproducir las condiciones dadas en la base económica y asegurar su existencia por medio de la violencia y/o la persuasión. Los primeros -los aparatos represivos del Estado, tales como la Policía, las Cortes, las prisiones, etc. trabajan por represión, mientras los últimos, los aparatos ideológicos del Estado, funcionan por ideología ${ }^{40}$. La educación es incluida aquí, junto con la religión, las leyes, la familia, las comunicaciones y la cultura (que en la opinión de Althusser se refiere a la literatura, las artes, los deportes, etc.).

La ideología, concebida como "una representación imaginaria de la realidad, como la forma mistificada en la cual los hombres experimentan su relación con el mundo" ${ }^{41}$ es utilizada para reproducir las relaciones de producción porque justifica su existencia como tal. Cada uno de los aparatos ideológicos del Estado contribuye a esta reproducción pasando a las nuevas generaciones la ideología de la clase dominante. En este proceso, Althusser enfatiza cómo la educación juega el papel dominante; ella proporciona a las masas la ideología que se acomoda al rol que ellas tienen que jugar en una sociedad de clases: el rol de explotado, de agente de la represión o de ideólogo profesional ${ }^{42}$. El rol dominante de la educación se debe principalmente a su eficacia. Como Althusser lo indica "ningún otro aparato ideológico del Estado tiene la audiencia obligatoria de la totalidad de los niños en una formación social capitalista". De otra parte, la escuela es la institución responsable de transmitir el conocimiento "envuelto en la masiva inculcación de la ideología" y esta es la manera como se reproducen las relaciones de explotación, en una sociedad capitalista ${ }^{43}$.

El papel dominante de la educación como aparato ideológico del Estado sirve para ilustrar la manera como una instancia de la superestructura, aunque en última instancia determinada por la base llega a convertirse en determinante por sí misma. Esto a su vez podría asumirse como el punto de partida para afirmar una relativa autonomía de la superestructura con respecto a la base. Pero esta autonomía no es suficiente para permitir que la educación tenga por lo menos cierto grado de independencia de la base económica y en consecuencia no da ninguna posibilidad para que la educación actúe sobre ésta y la transforme.

Con respecto a la educación permanente, las implicaciones de esta manera de pensar son claras. Si la educación es un resultado, una consecuencia de la base económica determinante, está condenada a reflejarla, justificarla y reproducirla. Esto es lo mismo que decir que en el sistema capitalista cualquier movimiento hacia la educación permanente inevitablemente terminará en la manipulación de las masas para apoyar el sistema. En la

\footnotetext{
${ }^{39}$ Idem., pág. 47 y 48.

${ }^{40}$ Idem. pág. 253.

${ }^{41}$ Sarup, M. Marxisxn and Education” pág. 151.

${ }^{42}$ Althuser, op. oit. pág. 260.

${ }^{43}$ Idem pág. 261.
} 
opinión de Furter ${ }^{44}$, "uno tiene que aprender más, no para vivir mejor, sino para producir más", de acuerdo con las necesidades del sistema económico. Una verdadera educación permanente es posible entonces únicamente en una sociedad que haya superado los obstáculos impuestos por el sistema capitalista, es decir, la división social del trabajo ${ }^{45}$ y la división de la sociedad en clases ${ }^{46}$. La situación para educadores progresistas y reformadores es, desde este punto de vista, desesperada.

\section{Interpretaciones alternativas}

Afortunadamente, la discusión no termina aquí. Las afirmaciones de Gelpi sobre la autonomía para la acción educativa en cualquier sociedad abren una nueva dimensión a los educadores y planificadores comprometidos con el cambio social. En sus propias palabras, la visión de educación permanente como algo que únicamente puede ser llevado a cabo en aquellas sociedades en las que un cambio revolucionario ha tenido lugar es "tan conservadora como aquella que excluye la dimensión política de la educación permanente" ${ }^{47}$. El enfoque de Althusser, aunque analiza acertadamente el carácter ideológico de la educación, deja sin solucionar el problema de cómo puede ocurrir el cambio en una sociedad. Su modelo es un modelo cerrado en sí mismo que no explica, finalmente, cómo se hace la historia

En consecuencia, la interpretación de Althusser debe ser considerada críticamente. Como Erber y Gleeson ${ }^{48}$ indican, su análisis "ignora el proceso que caracteriza a los hombres como productores activos de conocimiento y cambio social". Althusser no explica cómo los hombres adquieren el sentido de estructura social dentro de las relaciones de producción por consiguiente cómo se produce el conocimiento. Su modelo se aproxima más a la interpretación positivista del conocimiento como algo está fuera de la persona, manipulado por mecanismos y formaciones de su control. Aquí la crítica principal de Erber y Gleeson ${ }^{49}$ es que Althusser, tratando de explicar la manera, como se produce el conocimiento, termina describiendo cómo se consume. No es necesario decir que esto es lo mismo que negar la historia hecha por personas reales tanto en la base como en la superestructura. La relación dialéctica entre la escuela y la estructura social reside en "la conciencia de un continuo proceso de realización, a través de la acción en la intersección entre el sujeto condicionado y el mundo externo ${ }^{15}$.

Por otra parte, de acuerdo con Erber y Gleeson, Althusser fracasa en explicar por qué surgen los radicales y en entender la naturaleza de la rebeldía y de la duda radical. Enfatiza un modelo pasivo de socialización y niega la habilidad del hombre para tomar decisiones. Su teoría no deja espacio para una fuerza positivamente comprometida con la transformación social y en consecuencia ignora el hecho de que "la escuela (profesores y alumnos) puede representar, en situaciones reales, el espacio para un positivo interés en la transformación social" 50 . El análisis de Althuser no presta ninguna atención al proceso que se desarrolla en las escuelas y niega la posibilidad de una consideración crítica de problemas tales como indisciplina, bajo logro académico, y marginalidad, que pueden suscitar cuestionamientos tanto para el maestro como para los estudiantes respecto de una definición dada de su propia situación en la sociedad. En palabras de Erber y Gleeson los maestros y los estudiantes "están envueltos en una confrontación que no

\footnotetext{
${ }^{44}$ Citado por Ireland. op. cit. pág. 26.

${ }^{45}$ Vlnokur . A."Economic Analysis of Lifelong Education”. pág. 327.

${ }^{46}$ Suchodolski, B. " Lifelong Education. Some philosophical aspect” Citado por Dave, R.P. pág. 70.

${ }^{47}$ Gelpi, E. "A future for Lífeiong Education”. Vol. 1 pág. 11.

${ }^{48}$ Erber y Gleeson "Education as reproduction" pág. 78.

${ }^{49}$ Idem Pág. 79.

${ }^{50}$ Idem pág. 82.

Digitalizado por RED ACADEMICA
} 
depende enteramente de ellos, están localizados en relaciones antagónicas de lucha que... refleja sus respectivas posiciones dentro de las relaciones de producción. Y es aquí donde está el potencial para el cambio radical"51.

Así pues, contradicción y conciencia se convierten en elementos activos para el cambio. Y aquí la teoría marxista del conflicto debe ser tomada en consideración. Como Janne $^{52}$ lo indica, en el mundo capitalista la relación entre la base y la superestructura sirve para mantener el sistema. Pero la tensión orgánica entre partidos opuestos es el fenómeno más significativo. Desde una perspectiva marxista, en la medida en que la clase dominante desarrolle conciencia de su condición se organiza para cambiar las relaciones de producción y es así como se desarrolla la lucha de clases.

Aunque Janne está de acuerdo con la representación de la sociedad en términos de base y superestructura y con el carácter determinante de la base, él se refiere a la educación como uno de los campos en los cuales se desarrolla la lucha de clases. Y la lucha de clases es de acuerdo con Marx, el motor de la historia. Su análisis va más allá cuando él afirma, citando a Engels, que en ciertos momentos la superestructura puede convertirse en un agente de cambio de las relaciones de producción. Y como se dijo anteriormente, Althusser reconoce cierto grado de autonomía de la superestructura pero no hasta el punto de convertirla en un factor real de cambio.

El análisis marxista de Janne da una diferente dimensión al problema del determinismo cuando afirma que aunque en el largo plazo la historia se orienta en la dirección de un determinismo económico, hay en el corto plazo una interacción entre la base económica y la superestructura. Así, la lógica marxista misma es útil para considerar el rol de la educación como un factor de cambio. En el corto plazo la educación puede ayudar a "determinar las causas y consecuencias de cambios que han ocurrido, a planificar estrategias para cambios futuros en el cambio de la lucha social o para considerar los 'retardos culturales' creados por la tensión entre la fuerza de la tradición (rigidez de la superestructura) y los factores más fundamentales del cambio (la lógica de la contradicción)" ${ }^{\prime 3}$.

La función de la educación en el proceso del cambio social es entonces hacer explícitas, a través de la conciencia, las contradicciones existentes en la realidad social. Janne alcanza más o menos la misma conclusión de Erber y Gleeson aunque usando un diferente camino. El potencial de la educación como factor de cambio, y en consecuencia su autonomía en relación con la base, reside en su capacidad de hacer conscientes a las personas de los conflictos y de las contradicciones que existen en la sociedad.

Las críticas analizadas hasta ahora se centran únicamente en la noción de superestructura sin considerar la base. $Y$ es aquí donde el análisis de Williams es importante. De acuerdo con él, la proposición de una base determinada debe ser revaluada. En sus propias palabras:

"Tenemos que redefinir determinación en términos de establecer límites y de ejercer presión y no como algo predecible, prefigurado y controlado. Tenemos que redefinir superestructura en términos de un conjunto de prácticas culturales y no como algo reflejado, reproducido o dependiente. Pero sobre todo tenemos que redefinir la base no como una abstracción económica y tecnológica fija sino como la actividad específica de

\footnotetext{
${ }^{51}$ Idem, pág. 75.

${ }^{52}$ Jane, H.Theoretical foundations of Lifelong Education' en Dave, R. 1976.

${ }^{53}$ Janne, op. Cit pág. 143

Digitalizado por RED ACADEMICA
} 
hombres en condiciones sociales y económicas reales que contienen tradiciones fundamentales y variaciones y que está, en consecuencia en un continuo proceso dinámico"54

Lo que es nuevo en esta cita es la reformulación de la base como un proceso y no como un estado. La principal contradicción entre Williams y Althusser parece ser este concepto de la base como el área donde la sociedad se produce y reproduce ella misma - tanto producción material como reproducción de la vida real-y no únicamente como el área donde tiene lugar la producción primaria, dentro de los términos de la relación económica capitalista. Esta consideración nos conduce, siguiendo a Williams, "a estar menos tentados a despreciar o calificar como superestructural, y en ese sentido meramente secundario, ciertas fuerzas sociales vitales que son en el más amplio sentido de la palabra, desde su origen mismo, básicas" ${ }^{55}$.

Sin rechazar la noción de base y superestructura, Wílliams la califica de simplista por conducirnos a la conclusión de que la sociedad puede cambiar mucho más fácilmente de lo que en la práctica puede ser o ha sido. Prefiere entonces un modelo teórico que considera que cualquier sociedad en cualquier período particular de su historia tiene un sistema de valores, prácticas y significados, que no son abstractos - como se sugiere en el enfoque base - superestructuras pero que son experimentados por las personas y construidos dentro de su vida. Si lo que la gente aprende en la escuela, nos dice Williams, fueran únicamente significados y prácticas aislados de la clase dominante, que han sido impuestos en otros y que ocupan únicamente la parte superior de sus mentes, sería muy fácil eliminarlos y, en consecuencia, derrocar la cultura dominante ${ }^{56}$.

Así pues, debido a esta forma simplista y fragmentaria de representar la sociedad, la proposición de base y la superestructura plantea serios problemas teóricos. Un enfoque 'total' que se refiera a la sociedad como compuesta por un gran número de prácticas cuya interacción y combinación forman un todo social concreto sería más aceptable, aunque una noción de esta naturaleza puede conducirnos a ignorar el proceso de determinación y el concepto de intención social que son fundamentales para la comprensión de la teoría social de Marx. Porque las prácticas humanas en una sociedad dependen de la estructura y organización de esa sociedad específica y éstas están a su vez relacionadas con cierta intencionalidad social, esto es con las intenciones de la clase dirigente. El carácter determinado de muchas áreas de la ley social y política deben por lo tanto ser reconocidas con el fin de ver claramente el carácter de clase de la sociedad y ver cómo las leyes, las teorías e ideologías proclamadas como neutrales y universal-mente válidas expresan y ratifican la dominación de una clase particular. En consecuencia, un enfoque de totalidad puede ser utilizado únicamente si se lo combina con el concepto de hegemonía ${ }^{57}$.

Este concepto, tal como fue desarrollado por Gramsci, supone la existencia de algo "que es realmente total, que no es meramente secundario o superestructural como se entiende en el débil sentido de ideología, sino que es experimentado con tal profundidad que satura la sociedad hasta el punto de convertirse en el límite del sentido común para la mayoría de las personas que se encuentran bajo su dominio" ${ }^{\text {58 }}$. Hegemonía, en términos de Gramsci no se refiere ni al dominio de una nación sobre otra, ni a la dictadura de una clase. Se refiere al control de las clases subalternas por medio de instituciones culturales

\footnotetext{
${ }^{54}$ Williams, op. cit. pág. 203.

${ }^{55}$ Idem, pág. 204.

${ }^{56}$ Wiflianis, op. cit. pág. 204.

${ }^{57}$ Idem, pág. 205.

${ }^{58}$ Ibídem.

Digitalizado por RED ACADEMICA
} 
como la Iglesia, los sindicatos, la escuela, la prensa, etc. Dirección hegemónica es entonces "la forma de poder que da estabilidad y encuentra apoyo en un amplio consenso y aquiescencia"

Esto es así porque "cualquier relación hegemónica es necesariamente una relación pedagógica" 59

Para los fines de este artículo el concepto de hegemonía es el que da realismo a la función de la educación en el proceso de cambio social porque si bien es cierto que para una hegemonía existente es posible neutralizar o asimilar fuerzas culturales contra hegemónicas, la actividad militante, junto con un profundo cambio en la conciencia de las masas, puede conducir a su caída. En consecuencia un verdadero cambio revolucionario sólo se logrará si el ejercicio del poder por la clase trabajadora va de la mano con el establecimiento de una hegemonía de la clase trabajadora. Y esta es principalmente una empresa educativa a través de la cual los trabajadores aprendan a pensar y a actuar como una clase dirigente ${ }^{60 a " . ~ L a ~ e d u c a c i o ́ n ~ s e ~ c o n v i e r t e ~ e n t o n c e s ~ e n ~ u n a ~ p r a ́ c t i c a ~}$ revolucionaria y es aquí donde reside su verdadero carácter político.

\section{Educación permanente y formación profesional}

Desde esta perspectiva es importante analizar procesos de formación profesional, comúnmente vistos bien como parte del proceso de domesticación de la fuerza de trabajo y en consecuencia como un elemento clave para la reproducción de las relaciones de producción existentes, o simplemente ignorados por aquellos que asumen que hay una diferencia entre esta y la educación de los trabajadores y olvidan el rol de la primera en el proceso del cambio social.

Las instituciones de formación profesional han sido difícilmente consideradas como educativas en las sociedades capitalistas. Se ha dado por hecho que la educación es un bien en sí mismo, que tiene un valor intrínseco y que las actividades instrumentales o utilitarias están fuera del sistema educativo ${ }^{26}$. Este es el caso de la educación para el trabajo. Por su Valor extrínseco, la educación vocacional 'degenera' en mero entrenamiento y es en el mejor de los casos, un dispositivo para socializar a los jóvenes en la aceptación de los imperativos económicos y de las disciplinas del mundo del trabajo ${ }^{61}$. Esta visión de la educación vocacional está basada en una vi-Sión particular del trabajo, está basada en el supuesto de que el trabajo es un mal en sí mismo, únicamente justificado por su producto; una actividad que es externa al trabajador y que no le provee retribución distinta a satisfacer sus necesidades básicas.

Pese a las diferentes opiniones sobre este asunto, y particularmente del concepto marxista del trabajo como "la lucha con la naturaleza para crear la civilización" "62, la realidad ocupacional en las sociedades capitalistas confirma la anterior concepción del trabajo como una actividad deshumanizante. De acuerdo con Marx, en la sociedad industrial, basada en la división del trabajo, los trabajadores están condenados a hacer un trabajo que es externo a ellos, que no es parte de su naturaleza y que en consecuencia no los satisface sino que los niega como personas. Debido a las condiciones en las cuales se desarrolla el trabajo, los trabajadores no desarrollan su energía física y mental, sino que terminan exhaustos y mentalmente degradados. En la medida en que el trabajo no

\footnotetext{
${ }^{59}$ Gramsci, Quadermi, Vol. 2, Citado por Entwistle en “Education, work, and leisure”, pág. 12.

${ }^{60 a}$ Entwistle op. cit. pág. 13-14.

${ }^{26}$ Idem

${ }^{61}$ Idem, pág. 165.

${ }^{62}$ Entwistle, op. cit. pág. 145.

Digitalizado por RED ACADEMICA
} 
signifique la satisfacción de una necesidad, sino más bien el medio para satisfacer otras necesidades, no es una actividad voluntaria sino trabajo forzado cuyo producto ni siquiera pertenece al trabajador sino a alguien más ${ }^{63}$. Este es, en pocas palabras el concepto marxista de alienación y la razón por la cual el trabajo se ha convertido en 'la maldición de Adán'. En palabras de Marx: "y para el obrero que teje, hila, taladra, tornea, construye, cava, machaca piedras, carga, etc. por espacio de 12 horas al día, y ¿son estas 12 horas de tejer, hilar, taladrar, tornear, construir, cavar y machacar piedras la manifestación de su vida, su vida misma? Al contrario. Para él la vida comienza allí donde termina estas actividades, en la mesa de su casa, en el banco de la tabernas en la cama. Las 12 horas de trabajo no tienen para él sentido alguno en cuanto a tejer, hilar, taladrar, etc., sino solamente como medio para ganar el dinero que le permite sentarse a la mesa o en el banco de la taberna y meterse en la cama ${ }^{64}$

En términos prácticos hay dos maneras en las cuales esta cita puede ser interpretada. Una alternativa conformista, de una parte, toma el carácter deshumanizante del trabajo como dado y concibe el ocio como el residuo de tiempo de la jornada de trabajo durante el cual los hombres se involucran en aquellas actividades que les proporcionan satisfacción $^{65}$. Una perspectiva radical, de otra parte, el trabajo como esencial en la vida de las personas y como un medio para alcanzar realización siempre y cuando sean humanas las condiciones en las que se desarrolla. En otras palabras, mientras la primera interpretación piensa en el ocio como en el espacio en el cual la realización de la persona es posible, la última alternativa parece no hacer diferencia entre estas dos esferas y considerar el ocio como una continuación del trabajo, ambos en una visión humanízante. Así, mientras la primera ve las condiciones del trabajo como algo que no puede o no debe ser cambiado, la segunda implica un cambio revolucionario. Con respecto a la formación profesional, la primera la restringe al marco del entrenamiento de mano de obra mientras que la última la concibe como una parte fundamental de la educación de los trabajadores. Estas dos interpretaciones pueden ser comparadas con la distinción hecha por Parker ${ }^{66}$ entre los enfoques segmentalista y totalitarista del trabajo y del ocio, cuyas implicaciones políticas son enormes en relación no sólo con la educación vocacional sino también con la educación permanente y en consecuencia con el proceso del cambio social. Permítasenos analizar cada uno de estos enfoques en más detalle.

El enfoque segmentalista: diferenciación entre el trabajo y el ocio. Aunque algunas diferencias pueden encontrarse de un teórico a otro dentro de esta tendencia, todos ellos aparecen estar de acuerdo en que la segmentación del mundo del trabajo y del ocio es una característica del mundo moderno ${ }^{67}$

Trabajo y ocio son entidades autónomas y discontinuas cada una de las cuales existe a costa de la otra. El primero es un imperativo económico para proporcionar los requerimientos materiales de la vida; el último la compensación por haber tenido que vivir bajo 'la maldición de Adán ${ }^{68}$. De nuevo, esta afirmación puede ser interpretada de dos maneras dependiendo de si esta segmentación de esferas es concebida como deseable o como inevitable. En la primera posición, la diferenciación entre el trabajo y el ocio es vista como algo que estimula la innovación y la adaptación del individuo a la sociedad ${ }^{69}$,

\footnotetext{
${ }^{63}$ Entwistle, H. "Education, Work and Leisure pág. 18.

${ }^{64}$ Marx, K.”Trabaio asalariado y capital” pág. 68-69.

${ }^{65}$ Entwistle op. cit. pág. 2.

${ }^{66}$ Parker, S.”The future of work and leisure pág. 117-122.

${ }^{67}$ Idem, pág. 117.

${ }^{68}$ Entwlstie "Class, culture and education”, pág. 165.

${ }^{69}$ Duvin, citado por Parker, op. cit. pág. 117.

Digitalizado por RED ACADEMICA
} 
mientras que en el segundo caso se asume que diferenciación es inevitable y se sugiere que se introduzcan mejoras en cada una de las dos esferas. Como G. Friedman ${ }^{70}$, indica, "debe haber una revaluación del trabajo y debe haber oportunidades para la realización de los individuos y para su desarrollo en actividades fuera del trabajo". Friedman va más allá y acepta que "millones de trabajos no pueden ser revaluados" de manera que es necesario crear "un nuevo centro para el desarrollo humano en el uso activo del ocio". Desde esta perspectiva, la educación no debe estar relacionada con la preparación para el trabajo (entrenamiento) sino con la preparación para el ocio. Así, pues estos teóricos tienen poco que decir acerca del rol de la educación vocacional en el mejoramiento de la calidad de la vida. La automatización es vista como la única manera de liberar al hombre de la necesidad de trabajar, y a la escuela de su prostitución al sistema económico ${ }^{71}$.

Puede argumentarse sin embargo, desde un punto de vista progresista, que la educación para el ocio puede ser utilizada como un arma para desarrollar conciencia de las condiciones deshumanizante del trabajo y de la necesidad de cambiar las relaciones de producción existentes. En efecto, los reformadores pueden dejarse atraer fácilmente por la idea de emplear la educación para el ocio como un medio para inducir acontecimientos en la esfera del trabajo. En este punto de la discusión, las contribuciones de Marx son particularmente importantes. Como ya se dijo, aunque para Marx el trabajo es una actividad alienante en la sociedad capitalista, el ocio no representa un escape de esta situación infeliz ${ }^{72}$. Para él, el trabajo es una necesidad humana fundamental y como tal, determina la manera como se emplea el ocio. En otras palabras, la calidad del ocio, depende de la calidad del trabajo. Más aún la evidencia parece ponerse en contra de esta hipótesis de compensación. De acuerdo con Engels, es más posible que un trabajo mecánico, rutinario y en condiciones brutales sea compensado con la ebriedad en vez de con la búsqueda por 'horizontes más amplios'. En la actualidad muchos sociólogos están de acuerdo en que la industrialización empobrece la cultura popular y empuja a la clase trabajadora a buscar soluciones irracionales a los problemas de la vida ${ }^{73}$.

Trabajo y ocio no son independientes. Esta es una falsa diferenciación. Más aún, desde el mismo punto de vista capitalista, el ocio como tiempo libre, es decir libre de la supervisión del capital, plantea serios problemas al sistema en términos de reproducir una fuerza de trabajo disciplinada. La organización del ocio a través de la educación para el ocio y formas de consumo masivo, son los principales mecanismos que el sistema ha creado para asegurar la continuación de la necesaria disciplina del trabajo y por consiguiente de las existentes condiciones en que este se desarrolla ${ }^{74}$.

El enfoque segmentalista del trabajo y del ocio, es por tanto un enfoque esencialmente conservador; pretende que es posible mejorar la calidad de la vida, dejando intactas las causas de la infelicidad. Desde esta perspectiva, la educación para el ocio es un medio más para dominar a la clase trabajadora. Es el complemento perfecto para una educación vocacional concebida únicamente como entrenamiento.

\footnotetext{
${ }^{70}$ Friedman,en Parker, op. cit. pág. 118.

${ }^{71}$ Entwistle "Class, culture and education" pág. 165.

${ }^{72}$ Entwistle, H. "Education, work and ieisure", pág. 118.

${ }^{73}$ Entwistle, H. "Class, culture and education", pág. 176.

${ }^{74}$ Coalter y Barry "Leisure on the socioiogy of labour pág. 7. 


\section{Trabajo y ocio integrados: una visión totalitaria}

La visión alternativa es una visión revolucionaria. Toma como base la integración del trabajo y del ocio partiendo de la visión del trabajo como central al desarrollo humano. Nuevamente las contribuciones de Marx deben ser tomadas en cuenta, pues como se mencionó anteriormente, él no esta de acuerdo con la concepción del trabajo como un medio para satisfacer necesidades básicas tales como alimentación y vestido, sino como el proceso a través del cual los hombres moldean su entorno y determinan las circunstancias bajo las cuales quieren vivir. Así, pues, el trabajo es la manera en la cual cada uno de nosotros participa en la creación de nuestra propia civilización. Es fundamental para la vida social y es la fuente de toda la cultura humana, ya que el arte y el conocimiento científico emergen de los intentos humanos para entender, controlar y transformar el entorno ${ }^{75}$. En palabras de Marx: "El trabajo es en primer término, un proceso entre la naturaleza y el hombre, proceso que éste realiza, regula y con trola mediante su propia acción su intercambio de materias con la naturaleza. En este proceso, el hombre se enfrenta como un poder natural con la materia de la naturaleza. Pone en acción las fuerzas naturales que forman su corporeidad, los brazos y las piernas, la cabeza y la mano, para de ese modo asimilarse bajo una forma útil para su propia vida, las materias que la naturaleza le brinda. Y a la paz que de ese modo actúa sobre la naturaleza exterior a él y la transforma, transforma su propia naturaleza desarrollando las potencias que dormitan en él y sometiendo el juego de sus fuerzas a su propia disciplina

Visto de esta manera el trabajo no es solamente una actividad instrumental, es el proceso a través del cual la civilización se crea. En consecuencia, el ocio no es ni tiempo no consumido por el trabajo ni la condición en la cual éste se inmiscuye. Debe convertirse en un estilo de vida, si no en la vida misma, porque tiene un sentido en sí mismo ${ }^{77}$.

Por consiguiente, la sociedad no debe marginar el ocio sino integrarlo con el trabajo satisfactorio $^{78}$. Pero esta es una meta a largo plazo. Implica la transformación revolucionaria de las condiciones en las cuales el trabajo se desarrolla y esto únicamente es posible en una sociedad que ha llevado a cabo el cambio revolucionario de las estructuras socioeconómicas. No obstante, pueden darse algunos pasos en esa dirección y es aquí donde la formación profesional tiene una importante contribución para hacer al proceso del cambio social.

De acuerdo con Entwistle ${ }^{79}$ en términos ocupacionales deben distinguirse 3 niveles de competencia: la destreza en la ejecución de habilidades ocupacionales específicas, la capacidad para relacionarlas con niveles in' ustriales, comerciales y académicos en los cuales se practican y las actitudes que resultan de la percepción que tenga el trabajador de las dimen'iones políticas, sociales, morales y estéticas más amplias de su trabajo. Entrenamiento técnico y educación se refieren particularmente a las dos primeras áreas de competencia. La formación profesional las cobija pero incluye más que esto. Se refiere más a la tercera área de competencia, esto es, a la manera como las habilidades del trabajador se insertan en un contexto cultural más amplio. Esta connotación más amplia es la única que da a la educación vocacional una verdadera dimensión educativa y nos permite superar el angosto marco del entrenamiento.

\footnotetext{
${ }^{75}$ Entwistle "Education, work and leisure, pág. 25.

${ }^{76}$ Marx, K. "El Capital”, Vol. II., pág. 30.

${ }^{77}$ Entwistle, op. Cit., Pág. 168

${ }^{78}$ Parker, op. Cit., pág. 101

${ }^{79}$ Entwistle 'Class, cultura and education', pág.49-67

Digitalizado por RED ACADEMICA
} 
Aunque la situación ideal propuesta por Entwistle, de una educación vocacional integrada con la educación general, es difícil de lograr a través únicamente de los esfuerzos de educadores y planificadores involucrados en procesos de reforma educativa; algo puede hacerse en términos de integrar la formación profesional a la búsqueda de una nueva sociedad. Consecuentemente, si el concepto de la educación para el ocio deja intacto el problema de las condiciones de trabajo, es responsabilidad de una verdadera educación profesional permitir que el trabajador conozca cómo su ocupación y sus habilidades se relacionan con el contexto cultural, especialmente con la cultura política. En otras palabras formación profesional se define como la enseñanza y aprendizaje de competencias técnicas junto con la adquisición de una conciencia de las dimensiones socio-económicas del trabajo. Así pues, la educación vocacional es esencialmente una empresa política.

En su sentido más amplio, vocación significa el compromiso con un trabajo bien hecho. Implica una integración de las habilidades, el gusto personal, con cualidades como el sentido ciudadano y la moralidad. Desde este punto de vista, la educación profesional es auténtica experiencia educativa porque integra conocimiento, habilidades y actitudes que elevan la perspectiva del trabajador más allá de las demandas puramente técnicas impuestas por su trabajo. En una sociedad no revolucionaria donde las condiciones brutales del trabajo permanecen, así como la ignorancia sobre la naturaleza educativa de la formación profesional, los educadores deben impulsar el desarrollo de la conciencia de los alumnos en torno a la inutilidad de liberarse de la obligación de ganarse la vida, y más de la necesidad de comprender las implicaciones que para su trabajo tienen los procesos de toma de decisiones económicas y políticas y de tener un sentido de responsabilidad por la calidad de su trabajo ${ }^{80}$. La educación vocacional tiene que ver con la actividad esencial del hombre. Si el trabajo es considerado como la actividad a través de la cual la realización de la persona es posible, entonces la preparación para el trabajo debe ser llevada a cabo bajo este supuesto. En este sentido, educación para el trabajo es también educación para el ocio y parte del proceso hacia la educación permanente vista desde este punto de vista como la situación en la cual no hay división entre el ocio, la actividad productiva y el aprendizaje ${ }^{81}$

SUMARIO: Este artículo presenta una revisión crítica de las concepciones referentes a la educación como reproductora de las desigualdades sociales, al insistir en las potencialidades creativas de los seres humanos. Examina el problema de la reforma educativa permanente e intenta definir el rol del educador en sociedades como la nuestra.

DESCRIPTORES: Teoría de la educación, Educación no formal, Educación Permanente, Cambio social, Formación profesional.

\footnotetext{
${ }^{80}$ Entwistle, op. cit. pág. 179.

${ }^{81}$ Gelpi, E. 'Education and work', pág. 53-62.
} 


\section{REFERENCIAS}

ALTHUSSER, L. 1971 "Ideology and the Ideological State Apparatuses" (Ed.) Education, Structure and Society Harmondsworth, Penguin, 1972.

COALTER, F. And BARRY, N. 1982 "Leisure or the Sociology of Labour?" Polytechnic of Nortb

London Papera lii Leisure Studies No. 4.

DAVE, R.H. (Ed.) 1976 The Foundations of Lifelong Education Oxford, Pergamon Presa.

ENTWISTLE, H. 1970 Education, Work and Leisure London, Routledge asid Kegan Paul.

ENTWISTLE, H. 1979a Antonio Gramsci Conservative Schooling for Radical politice London, Routledge and Kegan Paul.

ENTWISTLE, H. 1979b Class, Culture and Education London, Methuen.

ERBER, M. asid GLEESON, D. 1977 "Education as Reproduction. A Critical Examination of sorne Aspects of the Work of Loui.s Althusser en Young M. and Whitty. G. (Eds.) Socíety, State and Schooling. Readings on the Possibilities for Education London, The Falmer Presa.

GELPI, E. 1980 A Future for Lifelong Education Vol. 1, Manchester, Umversity of Manchester Monographs.

GELPI, E. 1982 "Education and Work: Preiiminary Thoughts on the Encouragement of Productive Work lis the Educational Process" international Journal of lifelong Education Vol. 1 pp. $\sim 3-62$.

IRELAND, 7. 1978 Gelpi's View of Adult Educatton Manchester, University of Manchester Monographs.

JANNE, H. 1976 "Theoretical Foundations of Lifelong Education" en Dave, R.H. 1976.

MARX, C. 1971a El Capital Vol. 1, México Fondo de Cultura Económica.

MARX, C. 1971a "Trabajo asalariado y Capital en Marx. a. and Engels F. Obras escogidas Vol. I, Moscú, Ed. Progreso.

PARKER, S. 1971 The Future of Work and Leisure London, Paladln.

SARUP, M. 1978. "Marxism and Educatíon". London: Reoutledge, Kegan Paul.

SUCHODOLSKI, B. 1976 "Lifelong Educat.ion. Sorne Philosophicsl Aspects en Dave R.H. 1976.

VINOKUR, A. 1976 “Economic Analysis of Lifelong Education” en Dave, R.H. 1976.

WILLIAMS, R. 1976 "Base asid Superstructure lii Marxist Cultural Theory" en Dale, R. Schooling and Capitaliem London, The Open Unlversity Presa, Routledge asid Kegan Paul. 